\title{
In vivo and in vitro evidence of the neurotoxic effects of ropivacaine: The role of the Akt signaling pathway
}

\author{
ZHIHUA SUN ${ }^{1}$, HUINING LIU $^{2}$, QULIAN GUO ${ }^{1}$, XIAOPING XU ${ }^{1}$, ZHONG ZHANG $^{1}$ and NA WANG ${ }^{1}$ \\ Departments of ${ }^{1}$ Anesthesiology and ${ }^{2}$ Obstetrics and Gynecology, Xiangya Hospital, \\ Central South University, Changsha 410008, P.R. China
}

Received June 5, 2012; Accepted September 18, 2012

DOI: $10.3892 / \mathrm{mmr} .2012 .1115$

\begin{abstract}
There is a growing body of evidence that suggests common complications in regional anesthesia, including transient neurological syndrome, are caused by the neurotoxicity of local anesthetics (LAs). Ropivacaine is thought to be one of the safest LAs, however, there have been several studies detailing possible neurotoxic effects. At present, the exact molecular mechanism of ropivacaine-mediated neurotoxicity is unknown. The present study was designed to explore the possible mechanisms underlying the neurotoxicity of ropivacaine. The neurotoxic effects of ropivacaine were assessed in spinal cord by TUNEL staining for apoptosis and in cultured PC12 cells by cell viability assays. Protein kinase B (Akt) activation was evaluated by immunoblotting. Ropivacaine promoted apoptosis and caused cell death in a treatment group compared with a sham-operated group. Furthermore, ropivacaine significantly diminished Akt activation. There were significantly lower Akt levels in cells exposed to ropivacaine compared with controls. The present study demonstrated ropivacaine neurotoxicity in vivo and in vitro, mediated by the Akt signaling pathway. The neurotoxicity of apoptosis with concomitant cell death, mediated by ropivacaine, may offer an explanation for its adverse effects (e.g., transient neurological syndrome).
\end{abstract}

\section{Introduction}

Local anesthetics (LAs) have been used for relieving acute, intraoperative, postoperative and chronic pain for several years. Generally LAs are thought to be safe, but the neurotoxicity of LAs remains a considerable concern in certain cases. Ropivacaine is thought to be one of the safest LAs for pain relief, however, its efficiency is low, resulting in the utilization of high concentrations of LAs to relieve pain completely $(1,2)$.

Correspondence to: Dr Huining Liu, Department of Obstetrics and Gynecology, Xiangya Hospital, Central South University, No. 87 Xiangya Road, Changsha 410008, P.R. China

E-mail: sunjia201225@sina.com

Key words: local anesthetic, neurotoxicity, apoptosis, Akt, PC12 cells
Previous studies have reported considerable neurotoxicities associated with ropivacaine, particularly at high concentrations $(3,4)$. However, the molecular mechanisms through which LAs induce neurotoxicity remain poorly understood. Apoptosis, necrotic cell death and protein kinase B (Akt) signaling pathways may be involved (5-7).

It has been demonstrated that the activation of Akt is critical for cell anti-apoptosis (8). Survival factors suppress apoptosis by activating the serine/threonine kinase Akt, which then phosphorylates and inactivates components of the apoptotic process. Therefore, cells destined for apoptosis exhibit lower levels of Akt. Measuring Akt concentrations, under experimental conditions, may allow for an improved understanding of one of the possible mechanisms for cell death under specific conditions.

It previously has been reported (9) that high concentrations of ropivacaine result in neurotoxicity in specific cell lines. The current study was designed to determine the molecular neurotoxic mechanism of $1 \%$ ropivacaine in in vivo and in vitro models. In the present study, spinal cord in vivo and PC12 cell line in vitro models were utilized, with the aim to assess the possible neurotoxic cellular mechanistic effects of ropivacaine.

\section{Materials and methods}

Ethics and animals. The in vivo study was approved by the Ethics Committee for Animal Research of XiangYa Hospital in Central South University, China. Animal care and handling were in accordance with the Guide for the Care and Use of Laboratory Animals.

Chemicals. Commercially available ropivacaine (Naropin $10 \mathrm{mg} / \mathrm{ml}$, Astra-Zeneca, Rueil-Malmaison, Sweden) was used and diluted with $\mathrm{NaCl} 0.9 \%$.

Animal selection and housing. The experiment was performed using male Sprague-Dawley rats weighing 280-320 g. Rats were housed two/cage in the animal facility for one week, under 12:12 h light-dark cycle. Food and water were available at all times. Animal studies were performed following approval from the Institutional Animal Care and Use Committee in Xiangya Hospital, Central South University, China.

Surgical procedure for intrathecal catheterization. Rats were anesthetized with $10 \%$ chloral hydrate. As described 
previously (10), a sterile PE-10 tube filled with saline was inserted through the L5/L6 intervertebral space, placing the tip of the tube at the spinal lumbar enlargement level. The cannulated rats were observed for five days following intrathecal catheterization. Rats demonstrating symptoms of traumatic nerve injury or signs of neurological impairment during catheterization were excluded from further experiments.

Intrathecal administration of drugs. Following successful intrathecal catheterization, 72 rats were randomly divided into two groups (2x36). In group $\mathrm{R}$, rats received 8 injections of $1 \%$ ropivacaine $(0.12 \mathrm{ml} / \mathrm{kg})$, at 90 -min intervals, for a duration of $12 \mathrm{~h}$. Ropivacaine concentration was determined by a pilot study (11). A dosing interval of $1.5 \mathrm{~h}$ was based on a study by Rose et al (12). An identical amount of saline was administered by the same method in the group NS (control group). The catheter was flushed with $10 \mu \mathrm{l}$ of saline to account for the dead-space of the catheter. At each injection, the solution was administered over seconds and then capped with a pin. Each group was observed on days $1,3,5,7,14$ and $28(n=6$ at each time point).

Spinal cord section specimen. The animals in each group were sacrificed at each time point under terminal anesthesia. Transverse sections of the spinal lumbar enlargement region (L2-L6) in each animal were removed en bloc and each was dissected into two gross sections (A and B). The transverse specimen with spinal cord L3 (sample A) was used for TUNEL staining (see below) and embedded in paraffin. The posterior section specimen (sample B) was used for immunostaining examination.

TUNEL staining for apoptosis. For the detection of apoptosis, we used a Fluorescein FragEL ${ }^{\mathrm{TM}}$ DNA fragmentation detection kit QIA39 (Calbiochem, Darmstadt, Germany) to identify apoptotic nuclei in paraffin-embedded tissue sections. TUNEL was performed on 5- $\mu \mathrm{m}$ thick transverse sections of sample A. We used fluorescence microscopy and a x40 oil lens. Fields under x40 lenses within one section were randomly selected for quantification of TUNELpositive cells by counting. Data were expressed as average count/field/animal. Images were captured under high-power magnification (x200).

Western blot analysis. Sample B specimens from each group were homogenized in a lysis buffer containing protease inhibitors and phosphatase inhibitors. Supernatants were obtained following centrifugation at $10,000 \mathrm{x} \mathrm{g}\left(4^{\circ} \mathrm{C}, 5 \mathrm{~min}\right)$. Protein concentration was estimated using the Bradford reagent (Sigma, St. Louis, MO, USA). The protein was mixed with laemmli sample buffer and heated at $99^{\circ} \mathrm{C}$ for $5 \mathrm{~min}$. For western blot analysis, an equal amount of protein (50-80 $\mu \mathrm{g})$ was loaded into each well and subjected to $10 \%$ sodium dodecyl sulfate-polyacrylamide gel electrophoresis (SDS-PAGE). The separated proteins were then transferred from the gel to polyvinylidene fluoride (Millipore, Bedford, MA, USA) membranes and blocked in 5\% skimmed milk prepared in $1 \mathrm{X}$ TBST. Membranes were incubated with the following primary antibodies overnight at $4^{\circ} \mathrm{C}$ : anti-Akt (Santa Cruz Biotechnology, Inc., Santa Cruz, CA, USA), anti-phospho-Akt
(Stressgene, San Diego, CA, USA) or anti- $\beta$-actin (Santa Cruz Biotechnology, Inc.; each at 1:1,000). Blots were developed using Beyo ECL plus detection system (Beyotime Institute of Biotechnology, Shanghai, China) and relative band density was measured using FluroChem FC2 System (NatureGene Corp., Medford, NJ, USA).

Cell culture and treatments. PC12 cells (ProMab Bitechnologines Inc., Hunan, China) were routinely cultured in growth medium (GM) composed of DMEM and 10\% fetal bovine serum (FBS; Hyclone SH30084.03, Haikelong, China) at $37^{\circ} \mathrm{C}$ and $5 \% \mathrm{CO}_{2}$. The cells were pre-seeded at appropriate densities. Ropivacaine was prepared as stocks of $1 \%$ ( $15 \mathrm{mmol} / \mathrm{l}$ ) in GM with the $\mathrm{pH}$ adjusted to 7.4. The concentrations of ropivacaine used in the present study were based on dose-dependent neurotoxicity effects and on previous in vitro studies $(13,14)$. The concentrations of ropivacaine were $1,2.5$, $5,10,15$ and $25 \mathrm{mM}$. A high concentration of ropivacaine was included since it most likely causes cell death in PC12 cells. Each assay was observed at $48 \mathrm{~h}$.

MTT cell counting assay. The cell toxicity of ropivacaine was assessed using the 3-(4,5-dimethyl-thiszol-2-yl)-2, 5-diphenyl tetrazolium bromide (MTT) assay (Cayman Chemical Co., Ann Arbor, MI, USA). Cells were plated at 4,000 cells/well in 96-well plates and grown in GM in the absence (control group NS) or presence of ropivacaine (group R) for $48 \mathrm{~h}$. MTT assay was performed using an enzyme-linked immunosorbent assay, measuring optical density values at $570 \mathrm{~nm}(630 \mathrm{~nm}$ calibration).

Measurement of cell viability. Trypan blue exclusion assay was used to measure cell viability. Cells were cultured in GM in group NS or group R and plated at 20,000 cells/well in 24-well plates. Time and concentrations of ropivacaine were as described for the MTT assay. The medium was changed and images of the cells were captured daily with an inverted microscope (Olympus, Tokyo, Japan). Following image capture, cells were trypsinized and stained with trypan blue (Mediatech, Manassas, VA, USA). Viable (non-stained) and non-viable (blue) cells were counted using a hemocytometer (Beckman, Brea, CA, USA).

Cell western blot analysis. PC12 cells were grown in GM in group NS or group R for $48 \mathrm{~h}$. Cells were harvested and lysed in a lysis buffer containing protease and phosphatase inhibitors. Protein quantification, SDS-PAGE and immunoblotting were performed as described previously (15), using the following primary antibodies: anti-Akt and anti-phospho-Akt (Thr308; Cell Signaling, Danvers, MA, USA; each at 1:1,000) and anti- $\beta$-actin (Abcam, Cambridge, MA, USA; 1:5,000). Blots were developed and the relative band density was measured as described for sample B specimens.

Statistical analysis. Statistical data were from multiple experiments or measurements and were presented as the mean \pm SEM $(n=6)$. The significance of differences $(P<0.05)$ was evaluated by one-way ANOVA followed by the Bonferroni/Dunnett post hoc test, where appropriate. SPSS 18.0 (SPSS, Inc., Chicago, IL, USA) was utilized to perform the tests. 


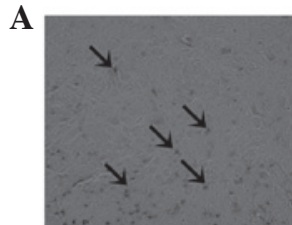

$1 d$

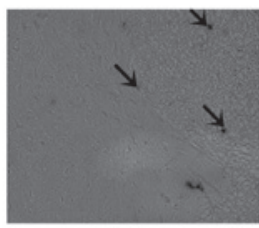

$7 d$

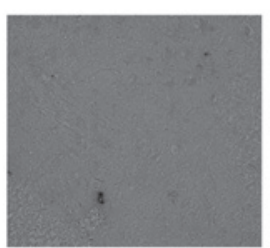

Control (NS)

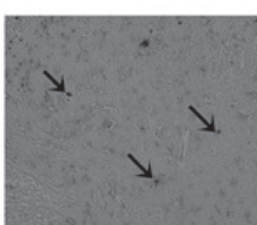

$3 d$
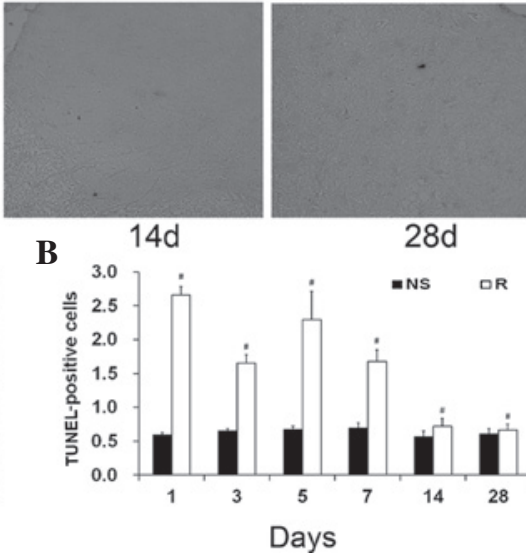

Figure 1. Effect of $1 \%$ ropivacaine on apoptosis in spinal cord. Group R, ropivacaine treatment; group NS, saline control treatment. (A) TUNEL staining was performed. Original magnification, $\mathrm{x} 200$; scale bar: $5 \mu \mathrm{m}$. (B) Analysis of apoptotic cells in spinal cord of groups R and NS at defined time points. ${ }^{*} \mathrm{P}<0.05$ vs. group NS.

\section{Results}

Surgical procedure. No visual evidence of CSF leakage was recorded following catheter placement or during the experiments. The rats demonstrated no signs of clinical complications of the central nervous system. Postmortem examination confirmed that all catheters were sited correctly.

Measurement of apoptosis - TUNEL staining. TUNEL staining was performed to determine whether exposure to $1 \%$ ropivacaine on days 1, 3, 5, 7, 14 and 28 triggers apoptosis-mediated neurotoxicity (Fig. 1). The percentages of TUNEL-positive cells in group NS were $0.030 \pm 0.005,0.032 \pm 0.008,0.034 \pm 0.007$ and $0.035 \pm 0.003 \%$ on days $1,3,5$ and 7 , respectively. The percentages of TUNEL-positive cells in group R were $1.33 \pm 0.26$, $0.89 \pm 0.15,1.15 \pm 0.18$ and $1.34 \pm 0.21 \%$ at the same time points. The mean number of TUNEL-positive cells in sample A of group R was significantly higher than that in group NS on days $1,3,5$ and $7(\mathrm{P}<0.05)$.

Ropivacaine and the Akt pathway. Akt activation was assessed by immunoblotting with an anti-activated Akt antibody (pAkt) in sample B, as described in Fig. 2A. Ropivacaine significantly suppressed Akt activation (Fig. 2A, lanes 1-6). Multiple experiments (n=6, Fig. 2B) demonstrated that the expression levels of pAkt in group $\mathrm{R}$ were significantly less than those in group NS at all time points $(\mathrm{P}<0.05)$. Total Akt levels were also examined by immunoblotting using an anti-total Akt antibody. We observed no significant difference of total Akt proteins in group R compared with group NS (Fig. 2C; $\mathrm{P}>0.05$ ). This correlated with the degree of apoptosis under the same conditions (compare Fig. 1B with Fig. 2A). Akt was activated in group NS cells (Fig. 3A, lane 1). By contrast, ropivacaine suppressed Akt activation (Fig. 3A, lane 2). Ropivacaine was associated with greater apoptosis in spinal cord and suppressed Akt activation.

Measurement of cell death - MTT assay. An MTT assay was performed to investigate the molecular mechanism(s) underlying this in vitro toxicity. To evaluate the toxic effects of ropivacaine, we performed cell counts using the culture model system PC12 (16,17). As shown in Fig. 4 by MTT assay, group NS cells grew vigorously and reached minimum confluence at $48 \mathrm{~h}$; the cell survival rate was $99 \%$. By contrast, the cell density in the presence of ropivacaine significantly declined; the cell survival rate was $46.1 \pm 7.3 \%$. Notably, group NS cells grew to a significantly higher density than those of group $\mathrm{R}(\mathrm{P}<0.05)$. These data demonstrate that ropivacaine leads to cell death.

Apoptosis and necrosis have been suggested to be two of the mechanisms by which LAs induce cell death (18). In the present study, we used trypan blue exclusion to assess the viability of PC12 cells. As shown in Fig. 5A, dead cells appear stained due to nuclear condensation. Viable cells were not stained. PC12 cells were grown in the absence or presence of ropivacaine. As already observed in Fig. 4, the total cell number in group $\mathrm{R}$ was significantly less than that of group NS (Fig. 5B). In group NS, dead cells (stained apoptotic and necrotic) were rare $(2 \pm 1 \%)$. In group R, the percentage of stained cells was $79.2 \pm 13.4 \%$ at $48 \mathrm{~h}$. Cell death percentages were calculated by normalizing non-viable cells to total cells and statistical results were exhibited (Fig. 5C). PC12 cells treated with ropivacaine had a had greater number of stained death identifiers.
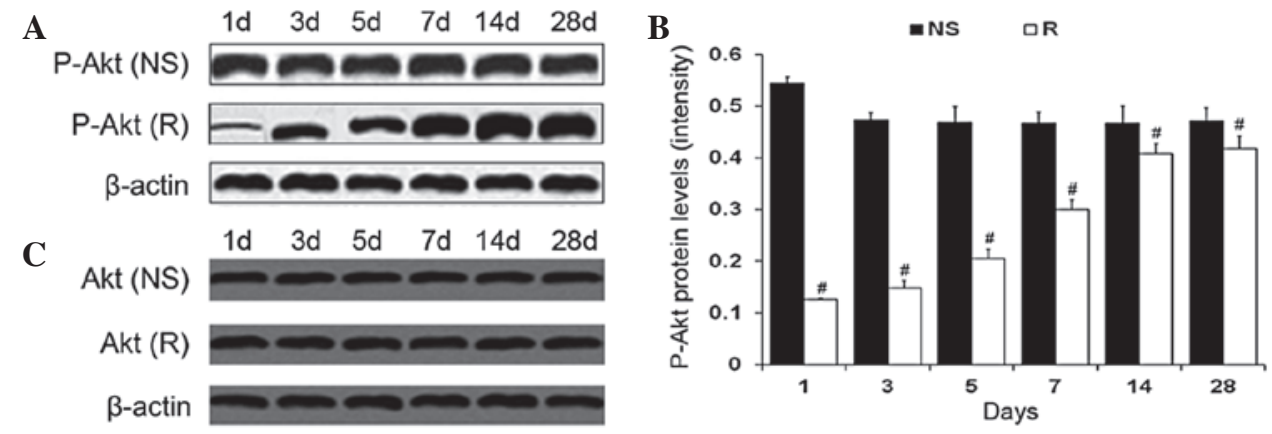

Figure 2. Effects of $1 \%$ ropivacaine on Akt activation. (A) Immunoblotting with anti-pAkt antibodies and (C) anti-total Akt antibodies. (B) Densitometric analysis of anti-pAkt signals from six independent experiments, ${ }^{\#} \mathrm{P}<0.05$. 
A

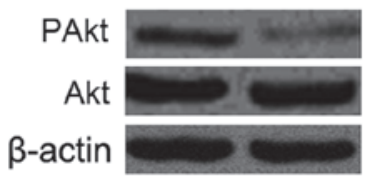

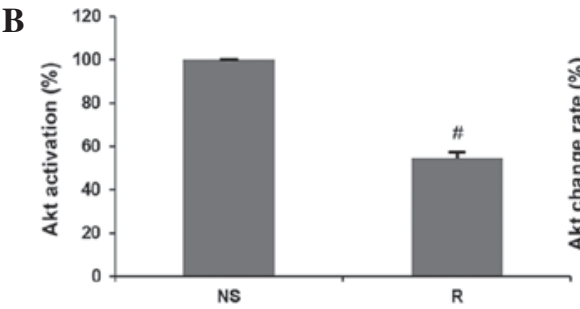

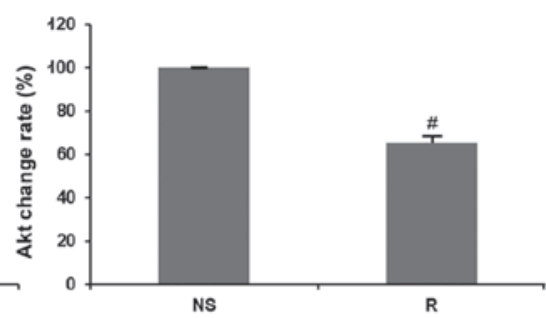

Figure 3. Effect of ropivacaine on Akt activation in PC12. Group R, ropivacaine treatment; group NS, saline control treatment. (A) Immunoblotting with anti-pAkt, anti-total Akt, and anti- $\beta$-actin antibodies, respectively. (B) Immunoblotting analysis of anti-pAkt signals (normalized to total Akt levels). Data are relative Akt activation levels and total Akt change with control set as $100 \%,{ }^{\circ} \mathrm{P}<0.05$.

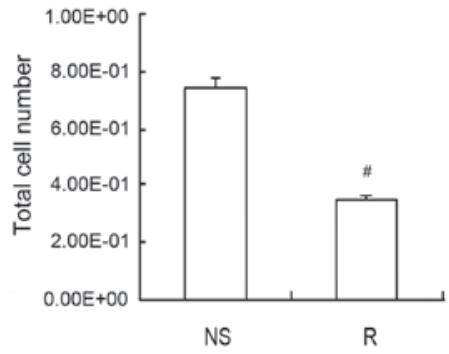

Figure 4. PC12 cells were exposed to ropivacaine for $48 \mathrm{~h}$ and then subjected to MTT assays. Group R, ropivacaine treatment; group NS, saline control treatment. " $\mathrm{P}<0.05$ vs. group NS.

$\mathbf{A}$
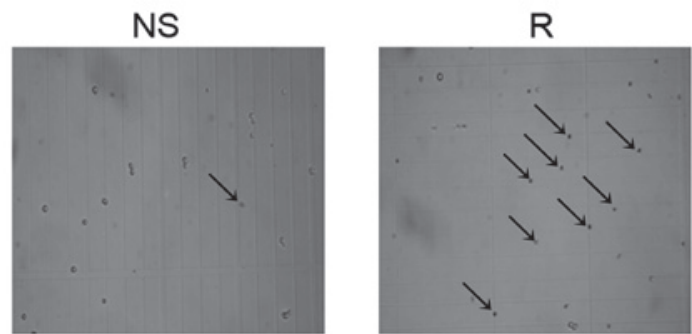

B

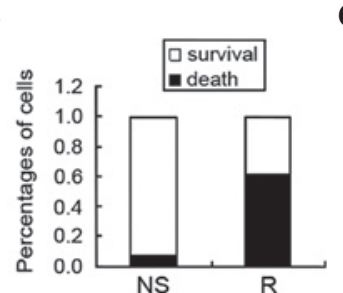

C

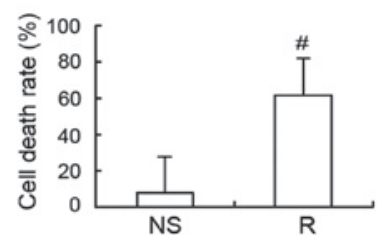

Figure 5. Toxicity of ropivacaine in vitro. Group R, ropivacaine treatment; group NS, saline control treatment. (A) Trypan blue assay. (B) Percentages of viable and dead cells from total cells. (C) Cell death rate (\%) was normalized to the group NS. ${ }^{\#}<0.05$ vs. group NS.

\section{Discussion}

Ropivacaine is available for spinal or intrathecal use and its use in clinical practice is common. The active duration of ropivacaine is 1.6-6 $\mathrm{h}$ and is considered a long-acting LA (19). Ropivacaine has numerous advantages for epidural analgesia compared with traditional medicines, including bupivacaine (20-22). However, in vitro and in vivo studies suggest that ropivacaine exhibits neurotoxicity $(11,14)$. In a previous study, we reported that following 6- or 48-h exposure during continuous spinal anesthesia, ropivacaine induced mild neuronal injury to the spinal cord and nerve roots (11). However, detailed molecular mechanisms, particularly the signaling pathways, by which the LAs exert neurotoxicity remain to be explored. The aim of the present study was to uncover the possible signaling pathways by which ropivacaine causes neurotoxicity. Numerous neurotoxicity studies have been conducted under various conditions, with exposure times ranging between $10 \mathrm{~min}$ and $48 \mathrm{~h}$. In this experiment, we used a rat model of repeated intrathecal administration of $1 \%$ ropivacaine for $12 \mathrm{~h}$ and examined the long-term (for 28 days) effects on lumbar enlargement spinal cord apoptosis following drug administration. The dosing interval, of $1.5 \mathrm{~h}$, was based on a study by Rose et al (12). We utilized the PC12 cell line and examined the toxic effects of $1 \%$ ropivacaine. Analysis of dose- and time-dependent effects in PC12 cells was based on a study conducted by Lirk et al (14). The present study focused on Akt, due to its critical signaling mode within cells under physiological and pathological cell survival mechanisms (8). In previous studies, Yuan et al suggested signaling pathways mediated by Akt are correlated to neuronal survival (23). In addition, Nakajima et al reported that persistent activation of Akt is important in neuronal cells (24).

In the present study, we demonstrated that ropivacaine exposure in vivo caused significant lumbar enlargement spinal cord apoptosis on days 1,3,5 and 7. In addition, we demonstrated that ropivacaine significantly inhibits cell growth and caused cell death following $48 \mathrm{~h}$ exposure in PC12 cells. Apoptosis is an essential process for cell integrity in development and survival. It is also triggered by physiological and non-physiological stimulation or insult, leading to pathological conditions. Although apoptosis is considered a major mechanism of LA-induced cell death, particularly in the case of neurotoxicity, LAs also induce necrotic cell death $(25,26)$. Our results indicate that apoptosis and cell death occur when ropivacaine is administrated intrathecally and exposed to PC12 cells. Our data suggest that therapeutic concentrations of the LA ropivacaine may be sufficient to cause local anesthetic-induced long term neurotoxicity. We also identified that Akt activation was decreased in the presence of intrathecal ropivacaine and in cultured PC12 cells exposed to ropivacaine. Inhibition of the Akt pathway may explain the resultant apoptosis upon exposure to ropivacaine. An inverse correlation between the levels of Akt activation and degree of apoptosis was noted in the current study. This supports the hypothesis that LAs, in particular ropivacaine, induce apoptosis via regulation of the Akt pathway. In the first 
part of this study, we assessed Akt activation by immunoblotting in repeated intrathecal ropivacaine. In the present study, we assessed Akt activation by immunoblotting with an antiactivated Akt antibody (pAkt) in PC12 cells (Fig. 2). When we examined total Akt levels by immunoblotting with an anti-total Akt antibody, we observed a significantly decreased level of total Akt proteins in group R cells compared with group NS (Fig. 3A). Compared with group NS, group R decreased Akt activity to $44.7 \pm 6.8 \%$. Total Akt was also decreased in group $\mathrm{R}$ to $33.2 \pm 8.5 \%$ compared with group NS. This largely correlated with the degree of dead cells under the same conditions (compare Fig. 3B with Fig. 4 or Fig. 5B/5C). Taken together, our data suggest that ropivacaine caused PC12 cell death, at least in part, via the inhibition of Akt activation. However, we cannot completely rule out other signaling pathway(s) as significant contributors to LA-induced toxicity. p38 MAPK and c-Jun $\mathrm{N}$-terminal kinase ( $\mathrm{JNK}$ ) pathways are known to be involved in ropivacaine-induced neurotoxicity $(11,27)$. These findings raise the possibility that certain, if not all, of these pathways may modulate specific LA-induced neurotoxicity independently or collectively. Further investigation is required to address these issues. Limited clinical studies have suggested that ropivacaine may lead to transient neurological syndromes $(28,29)$. The present study provides direct supportive evidence that in vitro and in vivo ropivacaine exposure results in neurotoxicity. The neurotoxicity of ropivacaine demonstrated in this study contributes to an emerging body of data supporting the detrimental neurotoxic effects of ropivacaine when used as an intrathecal anesthetic.

In conclusion, ropivacaine induces neurotoxicity in vivo and in vitro, and the Akt pathway is involved in the neurotoxicity of ropivacaine.

\section{Acknowledgements}

This study was supported by the Academician's Grant of Changsha (no. K0803154-31).

\section{References}

1. Malhotra N, Chanana C, Roy KK, Kumar S, Rewari V and Sharma JB: To compare the efficacy of two doses of intraperitoneal bupivacaine for pain relief after operative laparoscopy in gynecology. Arch Gynecol Obstet 276: 323-326, 2007.

2. Iida $\mathrm{H}$, Watanabe $\mathrm{Y}$, Dohi $\mathrm{S}$ and Ishiyama T: Direct effects of ropivacaine and bupivacaine on spinal pial vessels in canine. Assessment with closed spinal window technique. Anesthesiology 87: 75-81, 1997.

3. Lee SJ, Shin TJ, Kang IS, Ha JH, Lee SC and Kim HJ: AMPK attenuates bupivacaine-induced neurotoxicity. J Dent Res 89: 797-801, 2010.

4. Radwan IA, Saito S and Goto F: The neurotoxicity of local anesthetics on growing neurons: a comparative study of lidocaine bupivacaine, mepivacaine, and ropivacaine. Anesth Analg 94: 319-324, 2002

5. Maurice JM, Gan Y, Ma FX, Chang YC, Hibner M and Huang Y: Bupivacaine causes cytotoxicity in mouse $\mathrm{C} 2 \mathrm{C} 12$ myoblast cells: involvement of ERK and Akt signaling pathways. Acta Pharmacol Sin 31: 493-500, 2010.

6. Zink W and Graf BM: Local anesthetic myotoxicity. Reg Anesth Pain Med 29: 333-340, 2004

7. Zink W and Graf BM: The toxicity of local anesthetics: the place of ropivacaine and levobupivacaine. Curr Opin Anaesthesiol 21: 645-650, 2008

8. Manning BD and Cantley LC: AKT/PKB signaling: navigating downstream. Cell 129: 1261-1274, 2007.
9. Werdehausen R, Fazeli S, Braun S, et al: Apoptosis induction by different local anaesthetics in a neuroblastoma cell line. Br J Anaesth 103: 711-718, 2009.

10. Xu F, Li T and Zhang B: An improved method for protecting and fixing the lumbar catheters placed in the spinal subarachnoid space of rats. J Neurosci Methods 183: 114-118, 2009.

11. Zhong Z, Qulian G, Yuan Z, Wangyuan Z and Zhihua S: Repeated intrathecal administration of ropivacaine causes neurotoxicity in rats. Anaesth Intensive Care 37: 929-936, 2009.

12. Rose FX, Estebe JP, Ratajczak M, et al: Epidural, intrathecal pharmacokinetics, and intrathecal bioavailability of ropivacaine. Anesth Analg 105: 859-867, 2007.

13. Tan Z, Dohi S, Chen J, Banno Y and Nozawa Y: Involvement of the mitogen-activated protein kinase family in tetracaineinduced PC12 cell death. Anesthesiology 96: 1191-1201, 2002.

14. Lirk P, Haller I, Colvin HP, et al: In vitro, inhibition of mitogen-activated protein kinase pathways protects against bupivacaine- and ropivacaine-induced neurotoxicity. Anesth Analg 106: 1456-1464, 2008.

15. Huang Y, Li J, Zhang Y and Wu C: The roles of integrin-linked kinase in the regulation of myogenic differentiation. J Cell Biol 150: 861-872, 2000.

16. Greene LA and Tischler AS: Establishment of a noradrenergic clonal line of rat adrenal pheochromocytoma cells which respond to nerve growth factor. Proc Natl Acad Sci USA 73: 2424-2428, 1976.

17. Fasolato C, Zottini M, Clementi E, Zacchetti D, Meldolesi J and Pozzan T: Intracellular $\mathrm{Ca}^{2+}$ pools in $\mathrm{PC} 12$ cells. Three intracellular pools are distinguished by their turnover and mechanisms of $\mathrm{Ca}^{2+}$ accumulation, storage, and release. J Biol Chem 266: 20159-20167, 1991.

18. Boselli E, Duflo F, Debon R, et al: The induction of apoptosis by local anesthetics: a comparison between lidocaine and ropivacaine. Anesth Analg 96: 755-756, 2003.

19. McLure HA and Rubin AP: Review of local anaesthetic agents. Minerva Anestesiol 71: 59-74, 2005.

20. Wildsmith JA and Selander DE: Measuring the relative potencies of bupivacaine and ropivacaine in spinal anesthesia. Reg Anesth Pain Med 34: 73-74; author reply 734-735, 2009.

21. Guryay D, Karaege GT, Katircioglu K, Ozkalkanli MY, Ozgurbuz U and Savaci S: The effects of an epidural infusion of ropivacaine versus saline on sensory block after spinal anesthesia. Reg Anesth Pain Med 33: 217-221, 2008.

22. Michalek-Sauberer A, Kozek-Langenecker SA, Heinzl H, Deusch E and Chiari A: Median effective local anesthetic doses of plain bupivacaine and ropivacaine for spinal anesthesia administered via a spinal catheter for brachytherapy of the lower abdomen. Reg Anesth Pain Med 33: 4-9, 2008.

23. Yuan $Y$, Guo Q, Ye Z, Pingping X, Wang N and Song Z: Ischemic postconditioning protects brain from ischemia/reperfusion injury by attenuating endoplasmic reticulum stress-induced apoptosis through PI3K-Akt pathway. Brain Res 1367: 85-93, 2011.

24. Nakajima T, Iwabuchi S, Miyazaki H, et al: Preconditioning prevents ischemia-induced neuronal death through persistent Akt activation in the penumbra region of the rat brain. J Vet Med Sci 66: 521-527, 2004

25. Koike T, Tanaka S, Oda T and Ninomiya T: Sodium overload through voltage-dependent $\mathrm{Na}(+)$ channels induces necrosis and apoptosis of rat superior cervical ganglion cells in vitro. Brain Res Bull 51: 345-355, 2000.

26. Mukherjee PK, Marcheselli VL, Serhan CN and Bazan NG: Neuroprotectin D1: a docosahexaenoic acid-derived docosatriene protects human retinal pigment epithelial cells from oxidative stress. Proc Natl Acad Sci USA 101: 8491-8496, 2004.

27. Haller I, Hausott B, Tomaselli B, et al: Neurotoxicity of lidocaine involves specific activation of the p38 mitogen-activated protein kinase, but not extracellular signal-regulated or c-jun N-terminal kinases, and is mediated by arachidonic acid metabolites. Anesthesiology 105: 1024-1033, 2006.

28. Capdevila X, Pirat P, Bringuier S, et al: Continuous peripheral nerve blocks in hospital wards after orthopedic surgery: a multicenter prospective analysis of the quality of postoperative analgesia and complications in 1,416 patients. Anesthesiology 103: 1035-1045, 2005

29. Kitagawa N, Oda M and Totoki T: Possible mechanism of irreversible nerve injury caused by local anesthetics: detergent properties of local anesthetics and membrane disruption. Anesthesiology 100: 962-967, 2004. 